\title{
Electric Field Determination in DC Polymeric Power Cable in the Presence of Space Charge
}

\author{
W. Choo ${ }^{1}$ and G. Chen ${ }^{1}$ \\ ${ }^{1}$ University of Southampton, School of Electronics and Computer Science, Southampton SO17 1BJ, United Kingdom \\ *Email: ctc05r@ecs.soton.ac.uk
}

\begin{abstract}
The pulsed electroacoustic (PEA) technique was used to perform space charge measurements in polymeric power cables. However, for a practical dc power cable the electric field is affected by conductivity of the material, which is a function of both temperature and electric field. The coupled problems inflict difficulties to identify the electric field distribution in high voltage (HV) cables, which therefore poses threat to the reliability in operation of dc power cables.

In this paper we proposed a method of determining electric field distribution in XLPE power cable, where under temperature gradient the existence of space charge density of the cable is determined by means of a modified PEA system. Commercial $11 \mathrm{kV}$ ac XLPE power cable is applied and measured under an applied dc voltage of $80 \mathrm{kV}$. The space charge across the insulation was obtained and COMSOL Multiphysics software package is used to accurately determine the electric field distribution in the dc power cable by considering the influences of both the effects of temperature and electric field on the conductivity of the insulating material. Therefore, the results of the numerical modelling shall give us a clearer representation of the electric field distribution in HVDC cables.
\end{abstract}

\section{INTRODUCTION}

For the past few years, polymeric insulations for high voltage direct current (HVDC) applications have drawn a high level of interest and polymeric dc power cable is one of the areas that have drawn lots of attention recently. Knowing electric field distribution is an important issue in high voltage insulation design and comparing with its counterpart of ac power cable, one of the distinctive features is that the electric field distribution in a dielectric material under the application of dc voltage is determined by the conductivity of the insulating material. It is well known that the conductivity of an insulating material is a function of both the temperature and electric field. This means that the electric field will be consequently distorted depending on cable operation conditions. Therefore, the coupled problems inflict difficulties to identify the electric field distribution in HV cables. Consequently, there have been many attempts to characterise dc electric field distribution in polymeric materials [1]. On the other hand, it has been extensively reported that space charge can be easily formed in polymeric insulation materials under de conditions [2]. With the presence of space charge, the electric field will be modified. As a result, the conductivity varies with position, leading to change in electric field distribution, which therefore poses threat to the reliability in operation of dc power cables.

Following the progress made in non-destructive mapping of space charge distribution in polymeric materials, several techniques have been developed and applied not only to plaque samples but also on cable samples. At present, majority of the fundamental researches in space charge have been carried out on planar sample due to their easy manufacture and the influence of space charge on the electric field in the sample so far has been obtained without considering effect of both temperature and electric field on conductivity.

In this paper, space charge in a commercial $11 \mathrm{kV}$ ac XLPE power cable under $80 \mathrm{kV}$ dc electric stress was measured using the pulsed electroacoustic (PEA) technique [3]. A numerical simulation using COMSOL Multiphysics software package has also been adopted in this paper, which allows us to determine the electric field distribution of XLPE power cable in the presence of space charge considering the effect of conductivity under both temperature gradient and electric field.

\section{GENERAL EXPRESSION FOR DC CONDUCTIVITY}

Under the application of direct current (dc), the electric field is resistively graded. It is recognised that dc conductivity correlates strongly on both electric field and temperature [4]. The variation in conductivity with temperature of insulation has a major effect on field grading with load. It could be view that for a small temperature gradient, the electric field distribution is almost uniform. An increase of temperature gradient increases appreciably the degree to which the stress is inverted. However, for the field-dependence of the conductivity, it is normally considered to be a second order effect as the effect is relatively small.

Through the hopping theory model of conduction in dielectric [5], derivation of conductivity could be done 
from it. The expression of conductivity is well established and is expressed as

$$
\sigma(T, E)=A \exp \left(-\frac{\varphi q}{k T}\right) \frac{\sinh (B|E|)}{|E|}
$$

where $\mathrm{A}$ and $\mathrm{B}$ are constants, $\square$ the thermal activation energy in $\mathrm{eV}, \mathrm{q}$ the elementary charge, $\mathrm{T}$ the temperature in $\mathrm{K}, \mathrm{E}$ the electric field in $\mathrm{V} / \mathrm{m}$ and $\mathrm{k}$ the Bolzmann constant.

The above equation is applied in COMSOL to compute the electric field distribution in cables in the absence of space charge. It was examined that constants A, B and the thermal activation energy do varies depending on the materials and the conditions of it. Boggs et al [1] have demonstrated the parameters classification and classified it between two groups, good and bad dielectric respectively for XLPE cable.

\section{SPACE CHARGE IN POWER CABLE}

A section of an $11 \mathrm{kV}$ XLPE cable was prepared for space charge measurement. Space charge was measured using a modified PEA system [6] where a flat ground electrode was utilised instead of curved ground electrode for a conventional cable PEA system [7]. The major advantage of the modified PEA system is easy sample preparation. Compared with the plaque samples, obtaining space charge distribution from the PEA measurement for cable is more complicated and the detailed processes can be found in [6]. A voltage of +80 $\mathrm{kV}$ was applied to the central conductor of the cable, which has inner and outer radii of $8.75 \mathrm{~mm}$ and $11.55 \mathrm{~mm}$ respectively. The voltage was applied for 20 hours to generate sufficient space charge and the distribution was obtained immediately after the removal of the applied voltage as shown in Figure 1.

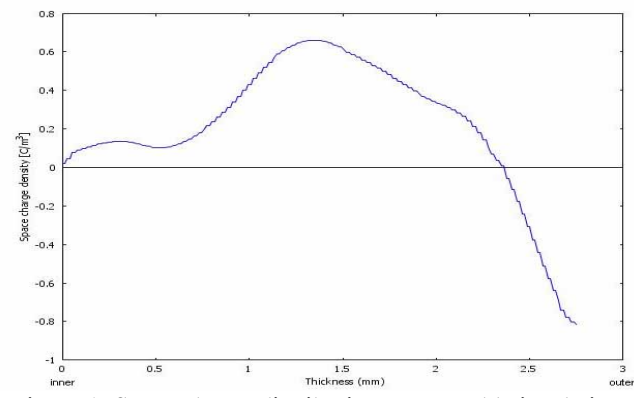

Figure 1: Space charge distribution across cable insulation.

It can be seen that significant amount of charges are accumulated in the vicinity of the two electrodes. Positive charge is formed adjacent to the inner electrode $(+80 \mathrm{kV})$ and negative charge close to the outer electrode, indicating that charge injection from the electrodes does take place, leading to homocharge formation. It has been reported that significant charge injection takes place when the applied electric field is greater than $25 \mathrm{kV} / \mathrm{mm}$ [8]. The applied electric fields in the present case are 32.8 and $24.9 \mathrm{kV} / \mathrm{mm}$ for the inner and outer electrodes respectively, therefore the injection is anticipated.

Experimental by $\mathrm{M}$. Fu also revealed that the space charge formed after 20 hours of stressing is very stable [9], implicating that the charges are deeply trapped.

Once the space charge distribution $\rho(r)$ is obtained in the sample material, the electric field $\mathrm{E}_{\mathrm{SC}}(\mathrm{r})$ produced by space charge can be calculated based on Poisson's equation represented by

$$
\frac{d E_{S C}(r)}{d r}+\frac{1}{r} E=\frac{\rho(r)}{\varepsilon_{o} \varepsilon_{r}}
$$

where $\mathrm{D}_{0}$ is the permittivity of vacuum and $\mathrm{D}_{\mathrm{r}}$ the relative permittivity of the material. Generally, it is believed that the field-dependence of the conductivity is relatively small. Therefore, the total field in the published work is obtained by summing the applied field and this field due to space charge and is expressed as

$$
E_{\text {Total }}=E_{\text {Applied }}+E_{S C}
$$

\section{SIMULATION RESULTS AND DISCUSSION}

In the present simulation, we have adopted the parameters for conductivity based on good dielectric material [1]. The parameters are input to COMSOL to accurately determine the electric field distribution in the presence of space charge. Three steps have been adopted. (i) Calculation of the electric field due to space charge based on Poisson's equation. (ii) The electric field obtained from (i) is then incorporated into conductivity equation to determine the electric field arisen from the applied voltage. (iii) Finally adding both the two fields together to form the total electric field indicated as in (3).

Figures 2 and 3 show the simulation results when $80 \mathrm{kV}$ is applied to the cable where no temperature gradient is considered. In figure $2 b$, it shows the conductivity is slightly linear as the conductivity decrease gradually from the inner to the outer electrode when space charge is ignored.

After considering the space charge field, figure $3 \mathrm{~b}$ shows that the conductivity of the material is no longer linear and the maximum conductivity reaches $1.25 \times 10^{-13}$ $\mathrm{S} / \mathrm{m}$. This maximum conductivity correlates to the maximum electric field produced by space charge. Subsequently, the electric field distribution due to applied voltage is greatly modified, showing in figure $3 \mathrm{c}$ the minimum occurs adjacent to the outer electrode. With the accumulated space charge, the voltage profile 
illustrated in $3 \mathrm{a}$ shows it starts to deviate to nonlinearity in the middle of the insulation.

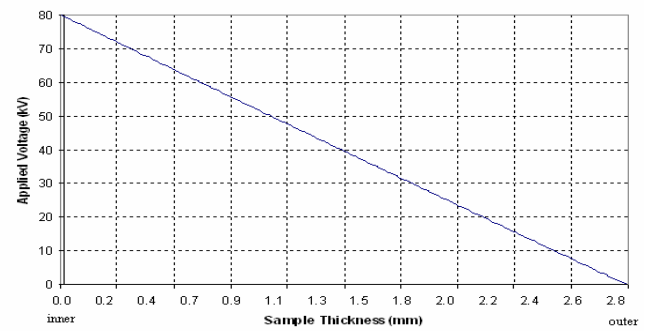

(a) Voltage distribution

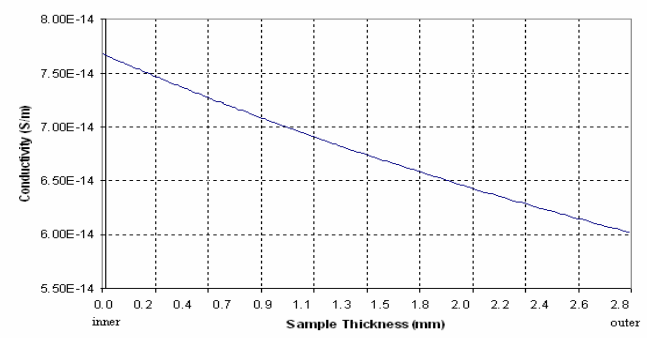

(b) Conductivity distribution

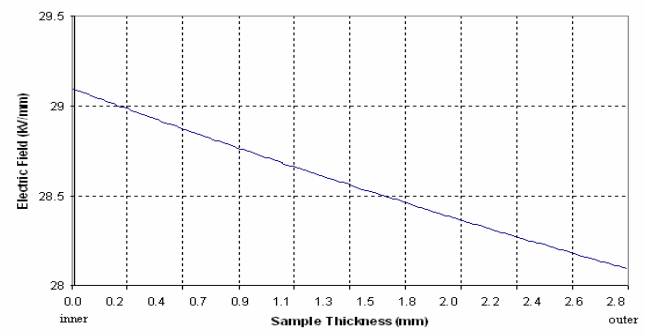

(c) Electric field distribution due to applied voltage

Figure 2: Simulation results considering the field-dependent conductivity when $0 \mathrm{~K}$ temperature gradient $T_{\text {inner }}=295 \mathrm{~K}$ and $T_{\text {outer }}=$ $295 \mathrm{~K}$ without the presence of homocharge.

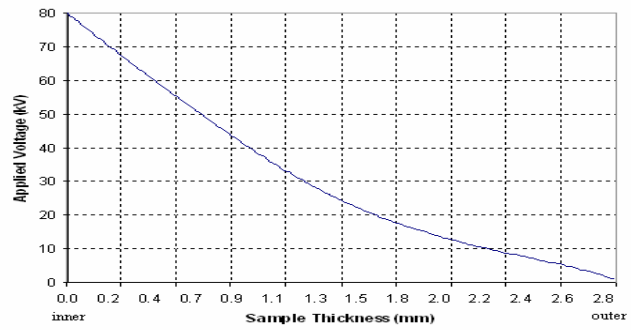

(a) Voltage distribution

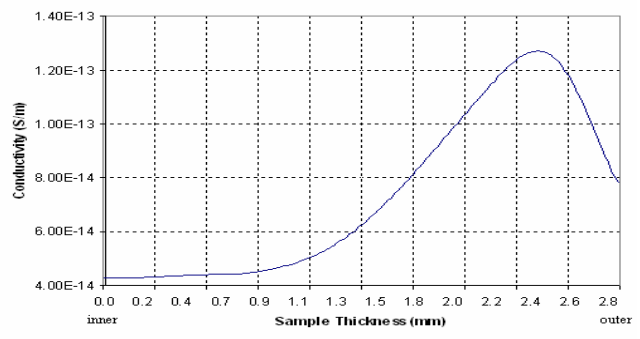

(b) Conductivity distribution

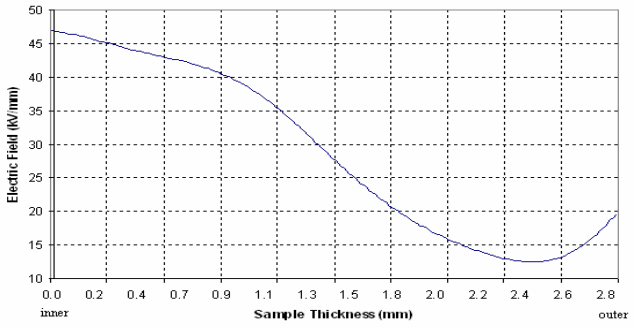

(c) Electric field distribution due to applied voltage

Figure 3: Simulation results considering the field-dependent conductivity when $0 \mathrm{~K}$ temperature gradient $\mathrm{T}_{\text {inner }}=295 \mathrm{~K}$ and $\mathrm{T}_{\text {outer }}=$ $295 \mathrm{~K}$ with the presence of homocharge.

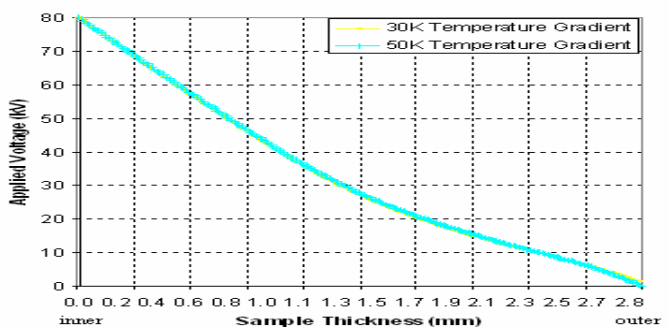

(a) Voltage distribution

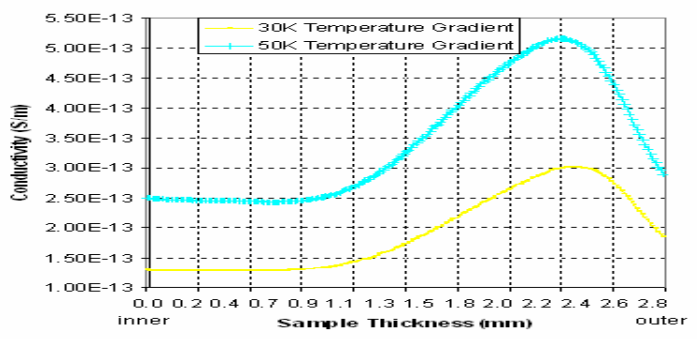

(b) Conductivity distribution

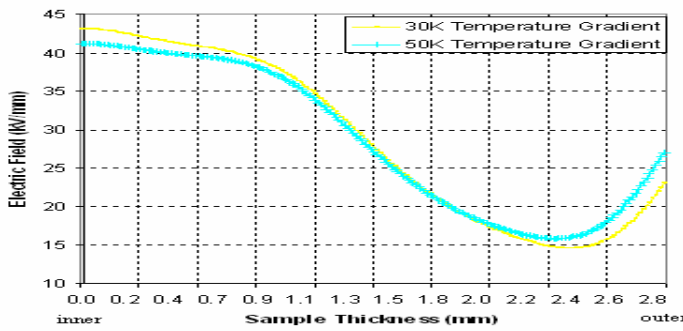

(c) Electric field distribution due to applied voltage

Figure 4: Simulation results considering both field and temperature gradient dependent on conductivity $\mathrm{T}_{\text {outer }}=295 \mathrm{~K}$.

The effects of temperature gradient are illustrated in figure 4. It shows a temperature gradient across the insulation will lead to an increase in conductivity distribution. At temperature gradient between $30 \mathrm{~K}$ and $50 \mathrm{~K}$, it shows that the electric field distribution due to applied voltage actually decrease at the inner region and tends to increase at the outer region of the insulation when the temperature gradient increases. 


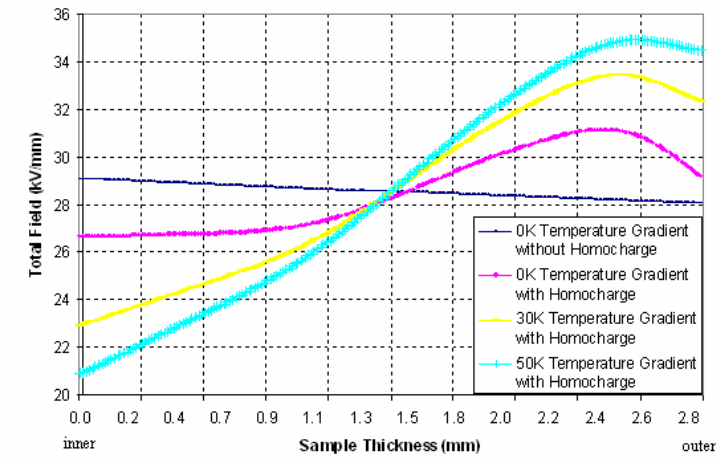

Figure 5: Total electric field distribution in cable with $\mathrm{T}_{\text {outer }}=295 \mathrm{~K}$.

Figure 5 illustrates the total electric field distribution in the cable's insulation. When the dependence of conductivity on temperature gradient and electric field are incorporated in the calculation, the stress in the inner region adjacent to the conductor is reduced. It can also be seen that as when the cable is heated, the maximum stress occurs at the outer region adjacent to the screen of the cable. When the temperature drop across the dielectric material increases, this maximum stress value adjacent to the screen of the cable also do increases.

In the present study, we have not considered the effect of using bad dielectric parameters and the influence of a temperature gradient on the electric field distribution in the presence of heterocharge formation. These studies will be presented soon in our future publications. More significantly, polarity reversal shall be investigated due to the production of significant field distortion.

\section{CONCLUSION}

We have measured space charge across the insulation in a dc power cable after an application of 10 $\mathrm{kV}$ for 20 hours using the PEA technique. Homocharge is accumulated in the XLPE dielectric material of the cable due to charge injection from the two electrodes.

As mentioned earlier, COMSOL Multiphysics software package is used to accurately determine the electric field distribution in the dc power cable by considering the influences of both the effects of temperature and electric field on the conductivity of the insulating material. The results indicate that when a cable is heated, the maximum stress occurs at the outer region adjacent to the screen of the cable and the value of this stress increases with the value of temperature drop across the dielectric material increases.

For these reasons, bulk space charge accumulation caused by non-uniform conductivity due to temperature gradient and electric field is therefore essential to both cable designers as well as the users.

\section{REFERENCES}

[1] S. Boggs, D. Damon, J. Hjerrild, J. Holbol and M. Henriksen, "Effect of Insulation Properties on the Field Grading of Solid Dielectric DC Cable.", IEEE TPD, Vol. 16, No.4, pp.456-461, 2001.

[2] G. Chen, "Space charge in solid dielectrics" ISH2005, Beijing, China, 2005.

[3] Y. Li, M. Yasuda and T Takada, "Pulsed electroacoustic method for measurement of charge accumulation in solid dielectrics", IEEE Trans. Dielectrics and Electrical Insulation, Vol.1, pp. 188 - 195, 1994.

[4] B. M. Weedy and D. Chu, "HVDC extruded cables - Parameters for determination of stresses, "IEEE Trans PAS. Vol. 103, pp. 662-667, 1984.

[5] A. R. Blythe and D. Bloor, Electrical properties of Polymers, $2^{\text {nd }}$ Edition, Cambridge University Press, 2005

[6] M. Fu and G. Chen, "Space charge measurement in polymer insulated power cables using flat ground electrode PEA system," IEE Proc.Science, Measurement \& Technology, Vol. 150, pp. 89-96, 2003.

[7] N. Hozumi, H. Suzuki, T. Okamoto, K. Watanabe and A. Watanabe, "Direct observation of timedependent space charge profiles in XLPE cable under high electric fields", IEEE Trans. Dielectr. Electr. Insul., Vol. 1, pp. 1068 - 1076, 1994.

[8] G. Chen and Z. Xu, "Space charge dynamics in low density polyethylene under dc electric fields", $12^{\text {th }}$ Intel Conf. on Electrostatics, Oxford, UK, 2007.

[9] M. Fu, "Space charge measurement in polymer insulated power cables using the PEA method", $\mathrm{PhD}$ thesis, University of Southampton, UK, 2002.

[10] J. C. Maxwell, A treatise on electricity and magnetism, Vol. I \& II, $3^{\text {rd }}$ edition, Claredon, Oxford, 1904.

[11] T. Tanaka and A. Greenwood, Advanced power cable technology, Vol. I, CRC Press, USA, 1983.

[12] P. H. F. Morshuis, R. Bodega, D, Fabiani, G. C. Montanari, L. A. Dissado, J. J. Smit, "Calculation and Measurement of Space Charge in MV-size Extruded Cables Systems under Load Conditions", Annual Report Conf. on Elec. Insul. and Dielectric Phenomenon, 2005 